\title{
Commensurability effects in the critical forces of a superconducting film with Kagomé pinning array at submatching fields
}

\author{
Nicolas P. Vizarim ${ }^{1}$, a , Maicon Carlone ${ }^{1}$, Lucas G. Verga ${ }^{2}$, and Pablo A. Venegas ${ }^{3}$ \\ 1 POSMAT - Programa de Pós-Graduação em Ciência e Tecnologia de Materiais, Faculdade de Ciências, \\ UNESP - Universidade Estadual Paulista, CP 473, 17033-360 Bauru, SP, Brazil \\ 2 Department of Chemistry, University of Southampton, Highfield, Southampton SO17 1BJ, UK \\ 3 Departamento de Física, Faculdade de Ciências, UNESP - Universidade Estadual Paulista, CP 473, 17033-360 Bauru, \\ SP, Brazil
}

Received 5 May 2017 / Received in final form 5 July 2017

Published online 13 September 2017 - C EDP Sciences, Società Italiana di Fisica, Springer-Verlag 2017

\begin{abstract}
Using molecular dynamics simulations, we find the commensurability force peaks in a twodimensional superconducting thin-film with a Kagomé pinning array. A transport force is applied in two mutually perpendicular directions, and the magnetic field is increased up to the first matching field. Usually the condition to have pronounced force peaks in systems with periodic pinning is associated to the rate between the applied magnetic field and the first matching field, it must be an integer or a rational fraction. Here, we show that another condition must be satisfied, the vortex ground state must be ordered. Our calculations show that the pinning size and strength may dramatically change the vortex ground state. Small pinning radius and high values of pinning strength may lead to disordered vortex configurations, which fade the critical force peaks. The critical forces show anisotropic behavior, but the same dependence on pinning strength and radius is observed for both driven force directions. Different to cases where the applied magnetic field is higher than the first matching field, here the depinning process begins with vortices weakly trapped on top of a pinning site and not with interstitial vortices. Our results are in good agreement with recent experimental results.
\end{abstract}

\section{Introduction}

When a transport current is applied in type II superconductors, vortices inside the material begin to move due to the Lorentz force, dissipating energy and destroying the zero-resistance state. In order to pin vortices and avoid their movement, pinning mechanisms have been introduced through artificial pinning centers (APCs) inside the material. These APCs work as attractive potentials for vortices, pinning them and enhancing the critical current density $J_{c}$.

During the last years, it has been made many efforts to understand the behavior of a vortex lattice under the influence of a periodic pinning array [1-37]. Nowadays, experiments on vortex dynamics with APCs have become possible thanks to the development of nanotechnology. Pinning arrays with different symmetries can be made by electron beam lithography (EBL), heavy ions bombardment or some substrate decorations [38]. These processes allow the formation of periodic and non-periodic pinning landscapes with the purpose of avoiding the vortex motion.

a e-mail: nicolasvizarim@gmail.com
It had been shown theoretically and experimentally that in the presence of a periodic and semi-periodic pinning lattice, vortices in superconductors show a variety of dynamic and commensurability effects, such as reduction of vortex mobility, change in the dynamic phases and increase of critical currents when compared to random arrays of APCs $[14,39,40]$.

In particular, for periodic pinning as, for example, square [12-15,35], triangular [3-7,34-37], rectangular $[1,22,28]$, honeycomb $[2,3,8,29,33]$ and Kagomé $[2,3,8,29-35]$, it is well accepted that for values of the external magnetic field $(B)$ higher than the first matching field $\left(B_{\phi}\right)$, vortices organize themselves in a highly symmetric way that leads to a perfect cancelation of the vortex-vortex interaction, generally forming a pattern which is commensurate with the pinning landscape, leading to pronounced peaks of critical force.

On the other hand, the vortex behavior under the influence of quasi-periodic pinning, as for example, Penrose [41-43], hyperbolic tessellation [44] and shield tiling [7] exhibit unusual commensurability effects enhancing the critical current for several values of applied magnetic fields [7,41-44]. Otherwise, in conformal pinning 
arrays, the critical currents do not change dramatically as a function of the applied field and exhibit higher depinning forces when compared to other pinning arrays [45-49], which is interesting for technological applications.

For the case of periodic pinning landscape, there are still open problems. For example, in type II superconducting thin films with Kagomé pinning lattices, both theoretical and experimental researches had been conducted focusing in the study of commensurability effects and critical forces $[2,3,8,29,33,35]$. Recent experimental work conducted by Cuppens et al. [8] investigated Al films with Kagomé pinning, where matching features were found at $B / B_{\phi}=1 / 3,2 / 3,1,4 / 3,5 / 3,2,2.2$ and 3 . However, previous theoretical simulations in superconducting films with the same pinning landscape did not predict commensurability peaks at any submatching field, arguing the absence of an ordered vortex lattice [2]. They only predicted pronounced peaks at most fields when $B / B_{\phi}=n / 3$, for $n>3$. Hence, the existence of critical force peaks when the external magnetic field is lower than the first matching field is still not clear. The usual commensurability condition, that $B / B_{\phi}$ must be an integer or a rational fraction, seems to be insufficient to have pronounced force peaks. The ordering of the vortex lattice is also important. Our calculations show that the ordering of the vortex ground state may strongly depend on the values of the pinning radius and strength.

The effect of the pinning strength had been investigated by several works $[2,3,5,14,25,37]$, but only few studied the effects of the pinning strength for $B / B_{\phi}<1$. Reichhardt and Grønbech-Jensen [5] studied the effect of the pinning strength for submatching fields in square and triangular pinning arrays, concluding that a stronger pinning potential may lead to the loss of pronounced critical force peaks for some values of external magnetic field. On the other hand, some experimental and theoretical works have investigated the influence of the pinning size in the critical current of superconducting films $[26,27,33,37,50]$, suggesting that a larger pinning radius lead to the enhance of the critical current due to vortex lattice ordering. However, I. Sadovskyy et al. [37] showed that the antidot should not become too large, because it may drop the critical current $J_{c}$ due to the reduced superconductivity gaps between the inclusions.

Recently, a simulation tried to search for the optimum pinning landscape in superconductors for all applied magnetic fields [51]. The idea was to model pinning size and density in order to enhance the critical current. But, they found that there is no universal optimum pinning for all magnetic fields, each application requires a different pinning landscape.

In the present work, we study the effect of the pinning strength and pinning radius in thin superconducting films, in order to better understand the commensurability effects in the critical forces for fractional matching fields. We also examine the depinning process and the role of interstitial vortices for $B / B_{\phi}<1$. The analysis of commensurability effects for the Kagomé pinning lattice at fractional matching fields has not been addressed.
The outline of the paper is the following. In Section 2, we present the model used for the simulation. In Section 3 we show the results for the vortex ground states and the critical forces as a function of the applied magnetic field, explaining the appearance of force peaks. In Section 4, we analyze the depinning process. In Section 5 we show the influence of pinning strength in the vortex ground states and critical forces. In Section 6 we analyze the influence of the pinning radius in the system with $B / B_{\phi}=1 / 3$. In Section 7 we present the conclusions of the work, highlighting the factors that influence the commensurability effects in the critical forces and in the depinning process for this type of system.

\section{Model}

In this simulation, the system considered is an infinite superconducting thin film, placed in the $x y$ plane with an external magnetic field $\vec{B}$ applied perpendicular to the film surface at zero temperature.

The dynamical properties of the two-dimensional system under consideration may be described by a set of Langevin equations [39]:

$$
\eta \frac{d \mathbf{r}_{i}(t)}{d t}=-\sum_{j \neq i} \nabla_{i} U_{v v}\left(\mathbf{r}_{i j}\right)-\sum_{p} \nabla_{i} U_{p}\left(\mathbf{r}_{i p}\right)+\mathbf{F}_{J}
$$

The first term in the right side of equation (1) is the vortex-vortex interaction in thin films, described by $U_{v v}\left(\mathbf{r}_{i j}\right)=-C_{v} \ln \left(\mathbf{r}_{i j} / \Lambda\right)[25,39]$, where $\mathbf{r}_{i j}=\left|\mathbf{r}_{j}-\mathbf{r}_{i}\right|$ is the distance between the vortex $i$ and $j$ and $C_{v}$ is defined as $C_{v}=\Phi_{0}^{2} / 8 \pi \Lambda$. The second term is the vortexpinning interaction which is described by $U_{p}\left(\mathbf{r}_{i p}\right)=$ $-C_{p} e^{-\left(\mathbf{r}_{i p} / a_{p}\right)^{2}}[39,40]$, where $\mathbf{r}_{i p}=\left|\mathbf{r}_{p}-\mathbf{r}_{i}\right|$ is the distance between the vortex $i$ and the pinning center $p, a_{p}$ is the pinning center radius and $C_{p}$ is the strength of the pinning potential. The third term is the driven force $\mathbf{F}_{J}=\left(\frac{d \Phi_{0}}{c}\right) \mathbf{J} \times \hat{z}$ due to the transport current $\mathbf{J}$. The summation of forces between particles, interacting logarithmically under periodic boundary conditions, is described by Grønbech-Jensen [52]. In the present case, the length scales are normalized by $4 \xi$, the time scale by $\tau=16 \eta \xi^{2} / C_{v}$ and the force scales by $C_{v} / 4 \xi$. Where $\xi$ is the coherence length, $\eta$ the Bardeen-Stephen friction [53], $\Phi_{0}$ the flux quantum, $\Lambda=2 \lambda_{L}^{2} / d$ the effective penetration depth, $d$ the sample thickness and $\lambda_{L}$ the London penetration depth $[39,40]$.

The calculations are made for the vortex system under the influence of Kagomé pinning array. The critical forces are calculated for transport forces applied in two mutually perpendicular directions, $x$ and $y$. The numerical simulations are made considering a fixed pinning density $n_{p}=N_{p} / L_{x} L_{y}=0.088 / \xi^{2} ; 126$ pinning centers were considered in the main simulation box, and $B / B_{\phi}=N_{v} / N_{p}$ was swept up to 1 , increasing $N_{v}$ by 6 . Using Generalized Simulated Annealing (GSA) minimization method [54], 


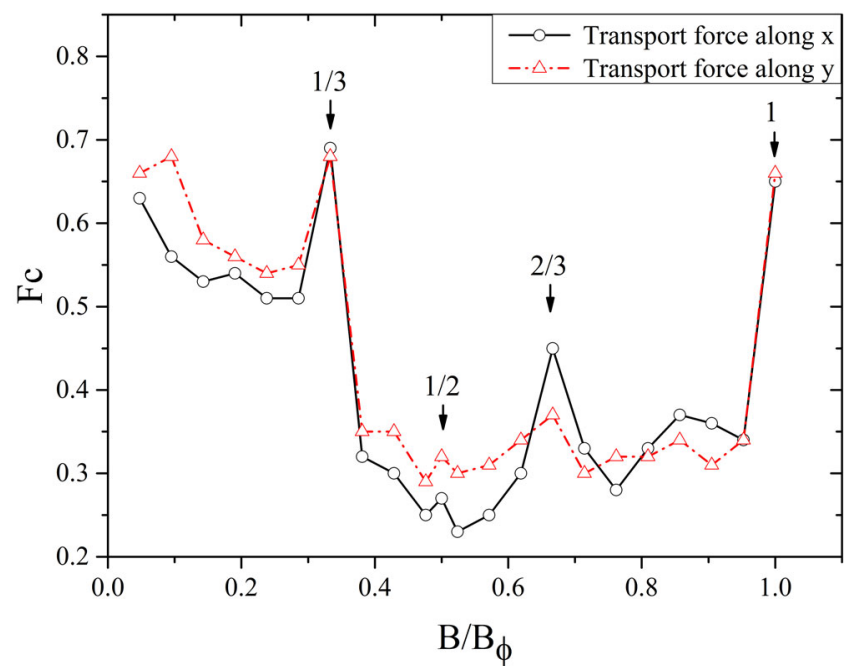

Fig. 1. Critical force $F_{c}$ vs. $B / B_{\phi}$ for the Kagomé pinning array with $C_{p}=0.2 C_{v}$ and $a_{p}=\xi$.

we obtain the lowest energy of the system and its associated vortex configuration. Through Molecular Dynamics technique, we integrate the set of Langevin equations and use the optimal vortex configuration as initial boundary value. Then, we proceed to increase the driving force to obtain their critical values. The driving force was increased slowly, in steps of $\Delta F_{j}=0.01$ until reaching values just above the critical force $F_{c}$. For each value of force, we use 30000 time steps for evaluation of the time averages. The calculation of critical forces requires the calculation of the time average vortex velocity $V=\left\langle V_{x}(t)\right\rangle=\left\langle\frac{1}{N_{\nu}} \sum_{i} \frac{d x_{i}}{d t}\right\rangle$ for $x$ direction and analogously for $y$ direction [39]. To determine these critical forces, in both $x$ and $y$ directions, the criterion used is that velocities $\left\langle V_{x, y}\right\rangle<0.001$ corresponds to vortex fluctuations around the equilibrium position.

\section{Vortex states and critical forces}

For the analysis of the vortex dynamics, it is necessary to search for the vortex ground state. Finding this ground state is a delicate work because there are several local minima with energy very close to the global minima, each of them associated to a different vortex array. As we are interest in commensurability effects, it is crucial to find the most stable vortex configuration to obtain the correct critical force. Using GSA, the energy of vortices under the influence of the Kagomé pinning array was minimized carefully for each value of $B / B_{\phi}$.

The integration of the set of Langevin equations were made for a transport force applied in both $x$ and $y$ directions. The results for the critical force, $F_{c}$, as a function of $B / B_{\phi}$ with $C_{p}=0.2 C_{v}$ and $a_{p}=\xi$, are plotted in Figure 1 . The results show pronounced critical force peaks at $B / B_{\phi}=1 / 3,2 / 3$ and 1 .

Figure 2 illustrates the vortex configurations obtained for the particular cases of $B / B_{\phi}=1 / 3$ and $2 / 3$. At

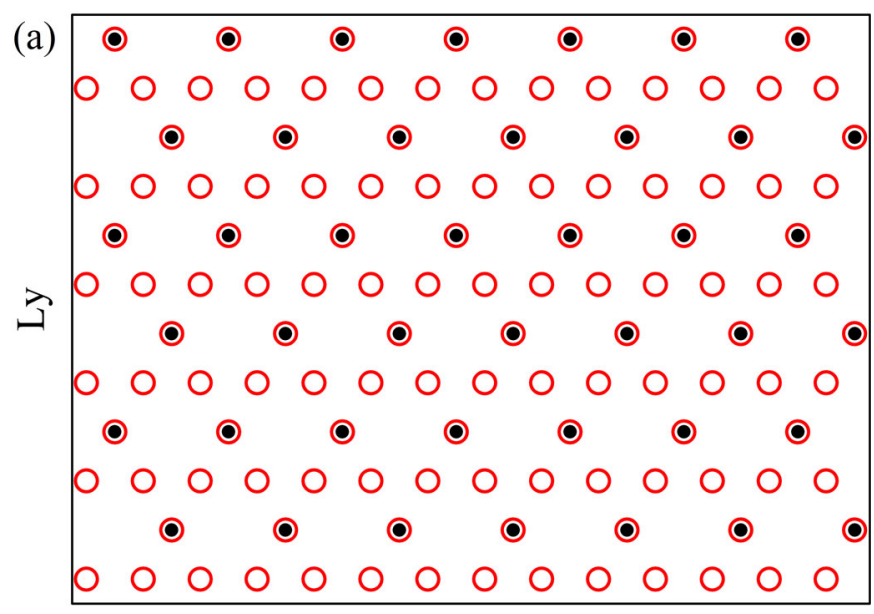

Lx

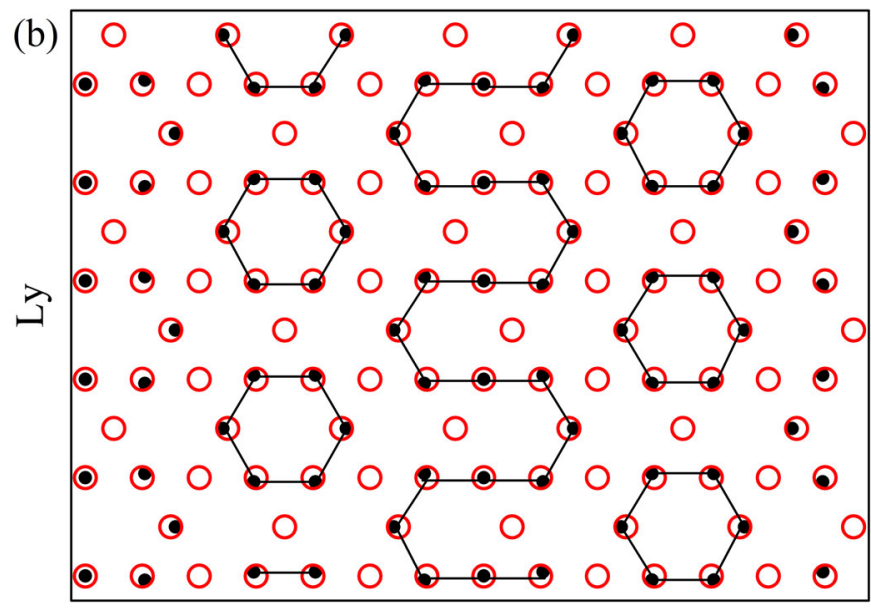

Lx

Fig. 2. Simulation box, where the black dots represent the vortex lattice and the red circles the Kagomé pinning lattice for (a) $B / B_{\phi}=1 / 3$, and (b) $B / B_{\phi}=2 / 3$, obtained from the minimization using $C_{p}=0.2 C_{v}$ and $a_{p}=\xi$. The lines at (b) are guides to the eye to identify the vortex sub-lattices.

$B / B_{\phi}=1 / 3$, our configuration (see Fig. 2a) matches the configuration predicted by Cuppens et al. [8], but for $B / B_{\phi}=2 / 3$, we found a vortex pattern with slightly lower energy than they proposed (see Fig. 2b).

The force peaks obtained at $1 / 3$ and $2 / 3$ agree with experimental results [8], but not with previous simulations [2], which proposed disordered vortex configuration and the absence of pronounced critical force peaks for any submatching field.

From Figure $2 \mathrm{a}$, it is easy to see that for $B / B_{\phi}=1 / 3$ the minimum energy configuration is a triangular vortex array, and from Figure $2 \mathrm{~b}$, we can see for $B / B_{\phi}=2 / 3$ an ordered lattice composed of two alternate sub-lattices, one with vortices at the vertices of a hexagon and the other forming a zigzag configuration. 


\section{Interstitial vortices and depinning process}

We also examined the role of interstitial vortices in the depinning process. The minimization of system energy at zero transport force shows that all vortices are seated at the top of a pinning site for all values of field, except for $B / B_{\phi}=1 / 2$ and $11 / 21$. Differently from cases with $B / B_{\phi}>1$, where interstitial vortices move before than the pinned ones $[3,12,14,15]$, our simulation shows that, in the present case, interstitial vortices are strongly pinned by the caged potential induced by other vortices, and the depinning process begins with vortices at the top of pinning centers. The equilibrium vortex positions are shown in the Figure $3 \mathrm{a}$ for $B / B_{\phi}=11 / 21$ with $C_{p}=0.2 C_{v}$ and $a_{p}=\xi$, where it is possible to see some interstitial vortices.

In Figure $3 \mathrm{~b}$ it is possible to see the depinning process just above the critical transport force, $F_{x}=0.23$. In this case, one vortex situated at the top of a pinning site, denoted by an arrow in that figure, begins to move. It is interesting to note that when the driven force is increased, but still under the critical force, some of the vortices change their original position, shown in Figure $3 \mathrm{a}$. This corresponds to transient vortex motions, i.e., the transport force induce vortex movements that reorder the system in a new static vortex state. A similar type of transient state had been observed before $[55,56]$.

We also performed the calculations using the transport force in the $y$ direction, and the result is shown in Figure 3c, where it is possible to see that the transient motion also occurs. In analogy to the case of transport force applied in $x$ direction, in $y$ direction, at $F_{y}=0.29$, a vortex (indicated by an arrow) seated at a pinning site begin to move, while interstitial vortices remain trapped.

\section{Pinning strength effect}

For a better understanding of the commensurability effects and the significance of the pinning strength for the critical forces, we performed simulations varying the pinning strength from $C_{p}=0.2 C_{v}$ to $C_{p}=0.8 C_{v}$, increasing the pinning strength by $0.1 C_{v}$. The calculations were made for transport forces applied in $x$ and $y$ directions.

The minimization process shows that for given values of $B / B_{\phi}$ the vortex ground state is different for different values of pinning strength. As an example, we compare the results for $C_{p}=0.8 C_{v}$ with $C_{p}=0.2 C_{v}$. The vortex arrangements for $B / B_{\phi}=11 / 21$ and $2 / 3$ using $C_{p}=0.8 C_{v}$ are shown in Figures $4 \mathrm{~b}$ and $4 \mathrm{c}$ and using $C_{p}=0.2 C_{v}$ in Figures $2 \mathrm{~b}$ and $3 \mathrm{a}$. For $B / B_{\phi}=11 / 21$, the interstitial vortices are not present anymore due to the increase in the pinning potential. That is, all vortices are trapped at the top of a pinning site stabilizing in a different configuration. This is in agreement with previous simulations with other pinning lattices that shows that for weaker pinning strength, vortices may be located in interstitial regions between pinning sites [5]. For the case of $B / B_{\phi}=2 / 3$, the vortex ground state also changes.

On the other hand, there are some vortex ground states that do not change as the pinning strength is increased.
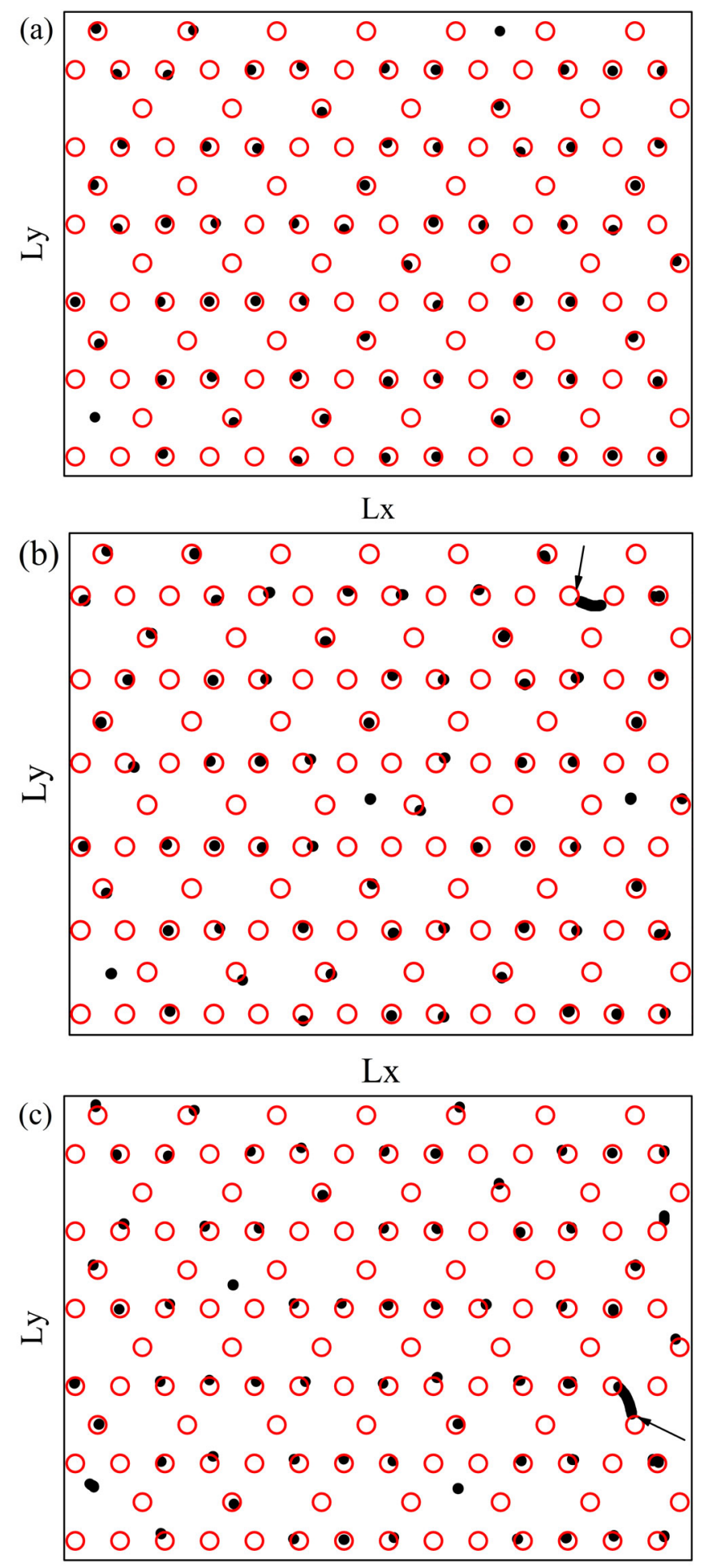

Lx

Fig. 3. (a) The simulation box with the vortices in the equilibrium position at $F_{x, y}=0$, where the black dots represent the vortices and the red circles the Kagomé pinning lattice, for $B / B_{\phi}=11 / 21, C_{p}=0.2 C_{v}$ and $a_{p}=\xi$. (b) and (c) The evolution of vortices just after their depinning process, for transport force $F_{x}=0.23$ and $F_{y}=0.29$ respectively, showing that the pinned vortices situated at the top of a pinning site (indicated by arrows) depin before, while interstitial vortices remain pinned. 
(a)

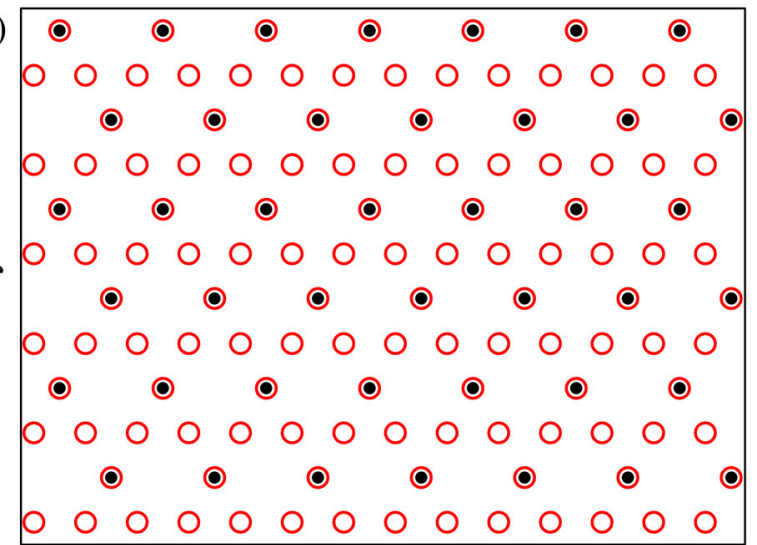

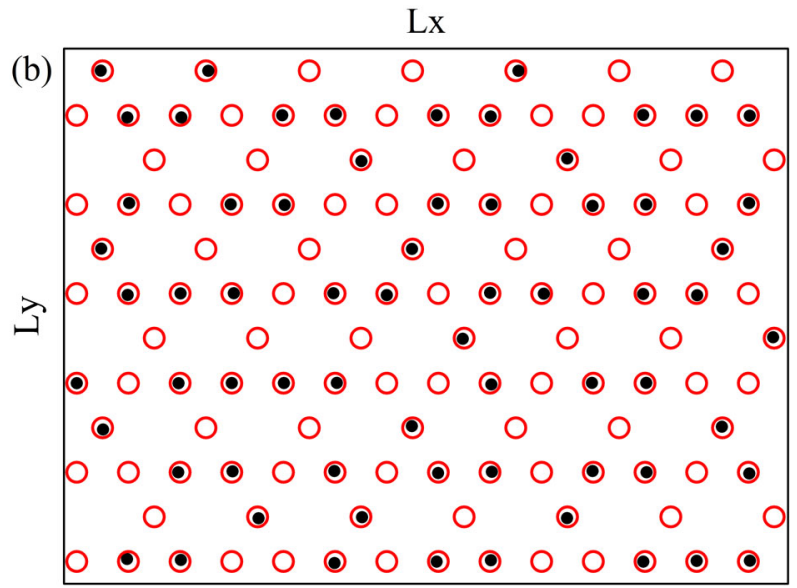



$\mathrm{Lx}$

Fig. 4. Simulation box, where the black dots represent the vortex lattice and the red circles the Kagomé pinning lattice for (a) $B / B_{\phi}=1 / 3$, (b) $B / B_{\phi}=11 / 21$ and (c) $B / B_{\phi}=2 / 3$, obtained from the minimization using $C_{p}=0.8 C_{v}$ and $a_{p}=\xi$.

Figures $2 \mathrm{a}$ and $4 \mathrm{a}$ show the vortex ground states for $B / B_{\phi}=1 / 3$ using $C_{p}=0.8 C_{v}$ and $C_{p}=0.2 C_{v}$, respectively. We can see that for both values of pinning strength the vortex ground state remains unchanged. In fact, our calculations show that the vortex ordering at this value of $B / B_{\phi}$ is maintained for all values of $C_{p}$. This also oc-
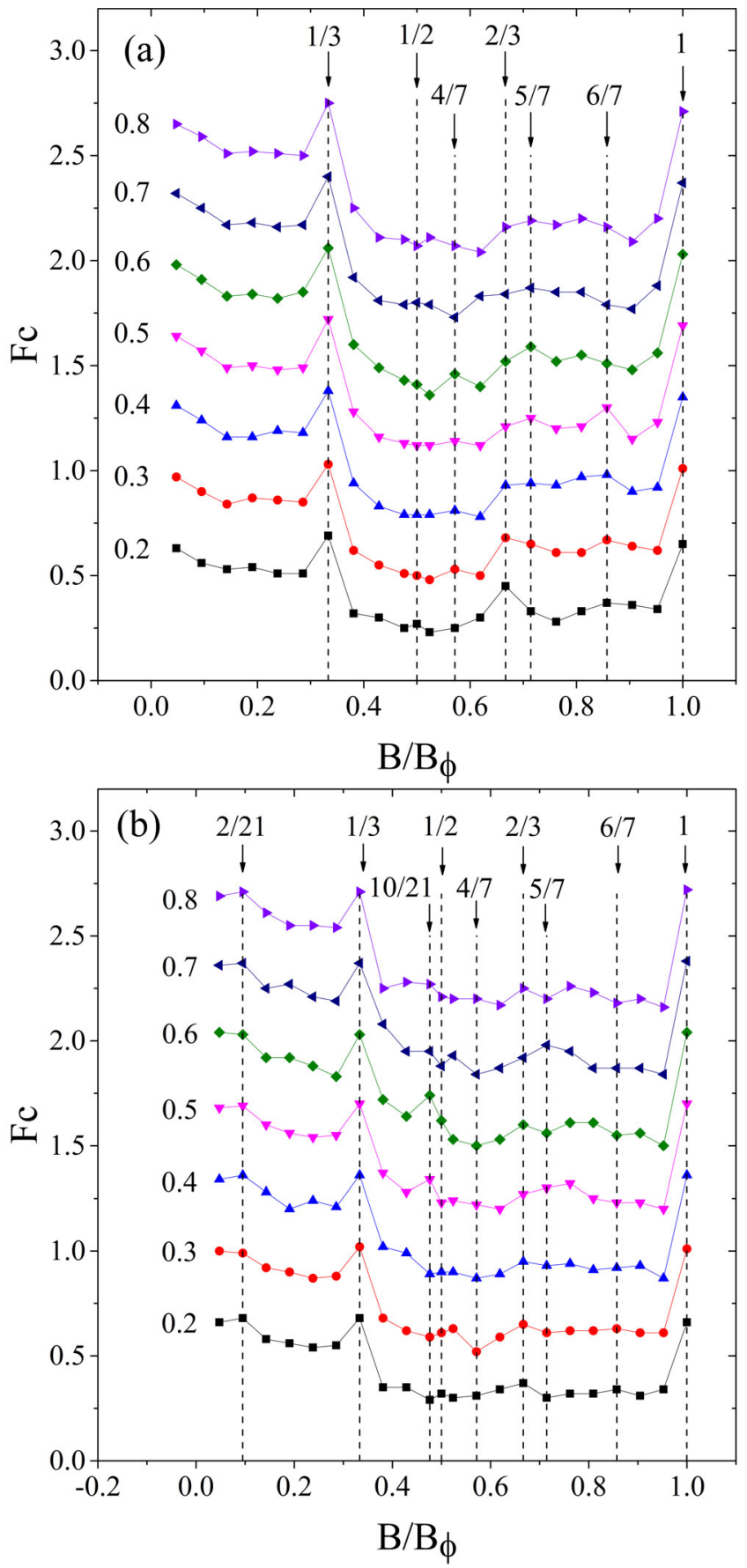

Fig. 5. Critical current $F_{c}$ vs. $B / B_{\phi}$ for the Kagomé pinning array with $a_{p}=\xi$ and varying $C_{p} / C_{v}$ from 0.2 to 0.8 . In (a) the transport force is applied in $x$ direction and (b) in $y$ direction.

curs for $B / B_{\phi}=1$, where the vortex lattice matches the Kagomé pinning lattice.

The results of the critical forces for both $x$ and $y$ directions as a function of the applied magnetic field for several values of pinning strength are plotted in Figure 5. The figure shows that the overall critical force values increase with the pinning strength and several peaks can be observed. We can see peaks at $B / B_{\phi}=2 / 21,1 / 3$, $10 / 21,1 / 2,2 / 3,4 / 7,5 / 7$ and 1 , depending on the direction 
of transport force and pinning strength value. The experiment [8] shows peaks of critical force at $B / B_{\phi}=1 / 3,2 / 3$ and 1 , which agree with our simulations for $C_{p}=0.2 C_{v}$ shown in Figure 1. However, it is clear from Figure 5 that changes in the pinning strength may influence the critical forces of the system, resulting in the fading of some force peaks due to the loss of the vortex lattice ordering. This loss of the vortex lattice ordering can be seen in Figure 4 when compared to Figure 2.

When the transport force is applied in $x$ direction, peaks of minor intensity at $B / B_{\phi}=1 / 2,4 / 7,5 / 7$ and $6 / 7$ can be seen in Figure 5 a depending on the pinning strength. Analogously, using force in $y$ direction, it is possible to see in Figure $5 \mathrm{~b}$ peaks for $B / B_{\phi}=2 / 21$ and $10 / 21$. These results show that the commensurability effects are different depending on the transport force direction, since some peaks are only present for $x$ direction and not for $y$, and vice-versa. Anisotropic behavior was previously observed in other theoretical and experimental works [3,16,21-23], but it is the first time that it is observed in Kagomé pinning lattice at submatching fields.

It is necessary to point out that the peaks at $B / B_{\phi}=$ $1 / 3$ and 1 (see Fig. 5) remain strong for both transport force directions and all values of pinning strength. This occurs because the ordering of the vortex lattice remain unchanged as the pinning strength is increased. However, other peaks became less evident as the pinning strength increases. This can be clearly seen for the case of $B / B_{\phi}=$ $2 / 3$ in Figure 5 a. For $C_{p}=0.2 C_{v}$, there was a critical force peak at this field, yet it faded for values of $C_{p} \geq 0.5 C_{v}$. Minor peaks were detected for $B / B_{\phi}=4 / 7,5 / 7$, and $6 / 7$, but they strongly depend on pinning strength. The reason for these peak fading is associated to the loss of ordering of the vortex ground state as the pinning strength is increased. Analogous behavior was found for the square and triangular pinning lattices $[5,25]$.

The simulation shows also a small peak at $B / B_{\phi}=$ $2 / 21$, which appears for most values of pinning strength, but the other minor peaks are pinning strength dependent. For example, the critical force peak for $B / B_{\phi}=1 / 2$ only exists when $C_{p}=0.2 C_{v}$, and the peak for $B / B_{\phi}=2 / 3$ exists only for some values of $C_{p}$. Differently from the case when the transport force is applied in $x$ direction, at $B / B_{\phi}=4 / 7,5 / 7$ and $6 / 7$ the critical force peaks are not evident in most of cases.

\section{Pinning radius effect}

As pointed before, critical force peaks at submatching fields $B / B_{\phi}=1 / 3$ and $2 / 3$ were observed experimentally [8] but not predicted in previous theoretical works [2]. To understand this discrepancy, we suggested two possible reasons: the pinning strength, and pinning center radius. In Section 5, it was shown that the increase in the pinning strength induce the fading of the critical force peak at $B / B_{\phi}=2 / 3$, however the peak at $1 / 3$ remains pronounced (see Figs. $5 \mathrm{a}$ and $5 \mathrm{~b}$ ). These results show that the disappearance of pronounced force peaks cannot be associated only to the pinning strength, it may also depends on

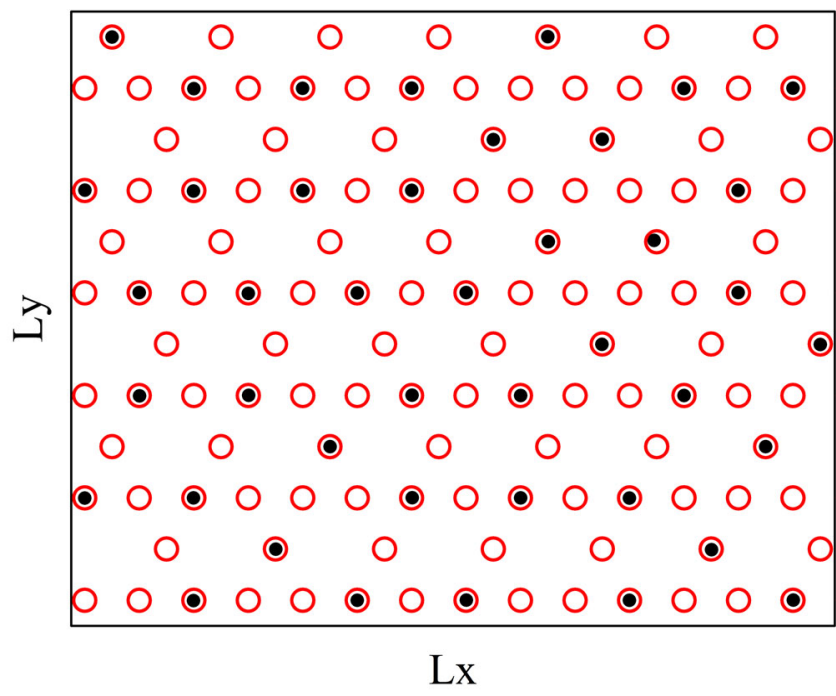

Fig. 6. Simulation box, where the black dots represent the vortex lattice and the red circles the Kagomé pinning lattice for $B / B_{\phi}=1 / 3, C_{p}=0.2 C_{v}$ and same $a_{p}$ used in reference [2].

the pinning size. Following the suggestion of experimental and theoretical results $[26,27,33,37,50]$, where their results showed that larger pinning radius may lead to the increase of the critical current, we used in our simulations a pinning radius close to the experimental value [8] and larger than that of reference [2]. As a result, vortices organize themselves in an ordered ground state (see Fig. 2a) provoking a pronounced force peak at this field for any value of pinning strength. Thus, to understand the absence of pronounced force peak at $B / B_{\phi}=1 / 3$, we performed a simulation using the smaller pinning radius of reference [2]. Our results show that instead of the ordered vortex configuration shown in Figure 2a, the smaller pinning radius induced the disordered vortex ground state illustrated in Figure 6. Despite all vortices are seated at a pinning site, they organized themselves in a disordered vortex arrangement weakening the pinning potential and preventing the existence of a pronounced critical force peak.

\section{Conclusions}

In this research, we numerically investigate the commensurability effects in the critical forces using a Kagomé pinning array at submatching fields. Our results show that critical force peaks do not only depend on the system being commensurate, that is, the rate between the external magnetic field and the matching field equal to integer or a rational fraction. We show that the order of the vortex ground state is also important. Our results show that weaker pinning strength and larger pinning radius lead to ordered vortex ground states and consequently to pronounced critical force peaks. Our results agree with previous experimental results of Cuppens et al. [8], where three main submatching features can be seen at $B / B_{\phi}=1 / 3$, $2 / 3$ and 1 , and elucidates why previous simulations [2] did 
not predict pronounced critical force peaks at submatching fields.

Our results show that the increase in the pinning strength changes the vortex ground state provoking the fading of pronounced force peaks for some values of field. It can be seen clearly that the peaks at $B / B_{\phi}=2 / 21$, $10 / 21,1 / 2,4 / 7,2 / 3,5 / 7$ and $6 / 7$ have fluctuating intensities as a function of the pinning strength. However, at $B / B_{\phi}=1 / 3$ and 1 , the peaks are always present. In these cases, the ordered vortex ground states do not change with pinning strength and the critical force peaks remain pronounced. Hence, the pinning strength is not sufficient to understand the disagreement afore mentioned, it is necessary to take into account the pinning radius effect.

The pinning size can change dramatically the vortex ground state. We analyzed the effect of pinning radius for the case of $B / B_{\phi}=1 / 3$, because at this field value the force peak remains pronounced for all values of pinning strength. Our results show that using the same pinning radius of reference [2], our vortex ground state also became disordered and no critical force peak is observed. We can conclude that larger APCs radius may lead to ordered vortex configurations, while smaller radius lead to disordered vortex configurations. Thus, the condition to have pronounced critical force peaks is not only associated to a commensurate vortex state, but it also requires to be ordered.

Otherwise, for the case of $B / B_{\phi}=1 / 3$, our vortex configuration matches Cuppens et al. prediction, but at $B / B_{\phi}=2 / 3$ our vortex ground state has slightly lower energy than that previously proposed by them.

The role of interstitial vortices was investigated for the present case, where $B / B_{\phi} \leqslant 1$. Our results show that interstitial vortices are present only when $B / B_{\phi}$ is around $1 / 2$ and for low values of $C_{p}$. Different from cases with $B / B_{\phi}>1$, where interstitial vortices depin before, our vortex dynamics simulations show that for the Kagomé pinning lattice at submatching fields, the depinning begins with vortices weakly pinned at the top of pinning sites, as shown in Figure 3.

Our work also showed anisotropic behavior when the transport force is applied in two mutually perpendicular directions, one along the $x$ direction and the other along $y$. For the case of $B / B_{\phi}=2 / 21$, there is a small critical force peak that only exists when the transport force is in $y$ direction, for most values of $C_{p}$. But for the case of $B / B_{\phi}=6 / 7$, the pronounced critical force peak only appears when the transport force is applied in the $x$ direction, for low values of $C_{p}$.

N.P.V. and M.C. acknowledges Capes (Brazil) for financial support and L.G.V. acknowledges the support from FAPESP (SP-Brazil) (Grant: 2012/22141-2), and from the Brazilian Government's Science Without Borders Programme (Grant: 206419/2014-7). This research was supported by the Center for Scientific Computing (NCC/GridUNESP) of the São Paulo State University (UNESP).

\section{Author contribution statement}

L.G.V., M.C., N.P.V., P.A.V developed the molecular dynamics algorithm used in this work. N.P.V. conducted the simulations. N.P.V., M.C. and L.G.V. worked on the analysis and elaborated the figures. N.P.V. and P.A.V. wrote the manuscript with input from all authors. P.A.V. was in charge of overall direction, planning and gave final approval of the version to be published.

\section{References}

1. C. Reichhardt, G.T. Zimányi, N. Grønbech-Jensen, Phys. Rev. B 64, 014501 (2001)

2. C. Reichhardt, C.J.O. Reichhardt, Phys. Rev. B 76, 064523 (2007)

3. C. Reichhardt, C.J. Olson Reichhardt, Phys. Rev. B 79, 134501 (2009)

4. C. Reichhardt, C.J. Olson, F. Nori, Phys. Rev. B 57, 7937 (1998)

5. C. Reichhardt, N. Grønbech-Jensen, Phys. Rev. B 63, $054510(2001)$

6. S. Ooi, T. Mochiku, K. Hirata, Phys. C: Supercond. 469, 1113 (2009)

7. D. Bothner, R. Seidl, V.R. Misko, R. Kleiner, D. Koelle, M. Kemmler, Supercond. Sci. Technol. 27, 065002 (2014)

8. J. Cuppens, G.W. Ataklti, W. Gillijns, J. Van de Vondel, V.V. Moshchalkov, A.V. Silhanek, J. Supercond. Nov. Magn. 24, 7 (2011)

9. A.N. Lykov, J. Low Temp. Phys. 164, 61 (2011)

10. L.G. Verga, M.M. Bonilha, M. Carlone, P.A. Venegas, J. Supercond. Nov. Magn. 26, 2147 (2012)

11. R. Simões, P. Venegas, D. Mello, J. Supercond. Nov. Magn. (2011)

12. K. Harada, O. Kamimura, H. Kasai, T. Matsuda, A. Tonomura, V.V. Moshchalkov, Science 274, 1167 (1996)

13. A.N. Grigorenko, G.D. Howells, S.J. Bending, J. Bekaert, M.J. Van Bael, L. Van Look, V.V. Moshchalkov, Y. Bruynseraede, G. Borghs, I.I. Kaya, R.A. Stradling, Phys. Rev. B 63, 052504 (2001)

14. C. Reichhardt, C.J. Olson, F. Nori, Phys. Rev. Lett. 78, 2648 (1997)

15. Q.-B. Ren, M.-B. Luo, Phys. Lett. A 377, 1966 (2013)

16. R. Cao, T.C. Wu, P.C. Kang, J.C. Wu, T.J. Yang, L. Horng, Solid State Commun. 143, 171 (2007)

17. M. Baert, V.V. Metlushko, R. Jonckheere, V.V. Moshchalkov, Y. Bruynseraede, Phys. Rev. Lett. 74, 3269 (1995)

18. A.N. Grigorenko, S.J. Bending, M.J. Van Bael, M. Lange, V.V. Moshchalkov, H. Fangohr, P.A.J. de Groot, Phys. Rev. Lett. 90, 237001 (2003)

19. S.B. Field, S.S. James, J. Barentine, V. Metlushko, G. Crabtree, H. Shtrikman, B. Ilic, S.R.J. Brueck, Phys. Rev. Lett. 88, 067003 (2002)

20. B.Y. Zhu, M. Liu, D.Y. Xing, B.R. Zhao, Z.X. Zhao, Phys. C: Supercond. 361, 107 (2001)

21. T.C. Wu, P.C. Kang, L. Horng, J.C. Wu, T.J. Yang, J. Appl. Phys. 95, 6696 (2004)

22. M. Velez, D. Jaque, J.I. Martín, M.I. Montero, I.K. Schuller, J.L. Vicent, Phys. Rev. B 65, 104511 (2002)

23. D. Jaque, E.M. González, J.I. Martin, J.V. Anguita, J.L. Vicent, Appl. Phys. Lett. 81, 2851 (2002) 
24. P.-J. Cuadra-Solís, A. García-Santiago, J.M. Hernandez, J. Tejada, J. Vanacken, V.V. Moshchalkov, Phys. Rev. B 89, 054517 (2014)

25. C. Reichhardt, G.T. Zimányi, R.T. Scalettar, A. Hoffmann, I.K. Schuller, Phys. Rev. B 64, 052503 (2001)

26. G.R. Berdiyorov, M.V. Milošević, F.M. Peeters, Phys. Rev. B 74, 174512 (2006)

27. G.R. Berdiyorov, M.V. Milošević, F.M. Peeters, Europhys. Lett. 74, 493 (2006)

28. M. Vélez, J.I. Martín, J.E. Villegas, A. Hoffmann, E.M. González, J.L. Vicent, I.K. Schuller, J. Magn. Magn. Mater. 320, 2547 (2008)

29. H. Shi-Kun, Z. Wei-Jun, W. Zhen-Chao, X. Hong, H. XiuFeng, G. Chang-Zhi, Q. Xiang-Gang, Chin. Phys. B 21, 087401 (2012)

30. M.F. Laguna, C.A. Balseiro, D. Domínguez, F. Nori, Phys. Rev. B 64, 104505 (2001)

31. R. Simões, P. Venegas, D. Mello, J. Supercond. Nov. Magn. (2011)

32. R.P. Simões, P.A. Venegas, D.F. Mello, J. Supercond. Nov. Magn. 26, 2277 (2013)

33. S.K. He, W.J. Zhang, H.F. Liu, G.M. Xue, B.H. Li, H. Xiao, Z.C. Wen, X.F. Han, S.P. Zhao, C.Z. Gu, X.G. Qiu, J. Phys.: Condens. Matter 24, 155702 (2012)

34. L.G. Verga, M.C. da Silva, R.P. Simões, D.F. Mello, P.A Venegas, J. Supercond. Nov. Magn. 26, 351 (2013)

35. D.J. Morgan, J.B. Ketterson, Phys. Rev. Lett. 80, 3614 (1998)

36. N.P. Vizarim, M. Carlone, L.G. Verga, P.A. Venegas, Mater. Res. (2017)

37. I.A. Sadovskyy, Y.L. Wang, Z.-L. Xiao, W.-K. Kwok, A. Glatz, Phys. Rev. B 95, 075303 (2017)

38. K. Matsumoto, P. Mele, Supercond. Sci. Technol. 23, 014001 (2010)

39. A.B. Kolton, D. Domínguez, N. Grønbech-Jensen, Phys. Rev. Lett. 83, 3061 (1999)
40. A.B. Kolton, R. Exartier, L.F. Cugliandolo, D. Domínguez, N. Grønbech-Jensen, Phys. Rev. Lett. 89, 227001 (2002)

41. V.R. Misko, S. Savel'ev, F. Nori, Phys. Rev. B 74, 024522 (2006)

42. R.B.G. Kramer, A.V. Silhanek, J. Van de Vondel, B. Raes, V.V. Moshchalkov, Phys. Rev. Lett. 103, 067007 (2009)

43. A.V. Silhanek, W. Gillijns, V.V. Moshchalkov, B.Y. Zhu, J. Moonens, L.H.A. Leunissen, Appl. Phys. Lett. 89, 152507 (2006)

44. V.R. Misko, F. Nori, Phys. Rev. B 85, 184506 (2012)

45. S. Guénon, Y.J. Rosen, A.C. Basaran, I.K. Schuller, Appl. Phys. Lett. 102, 252602 (2013)

46. C.J. Olson Reichhardt, Y.L. Wang, Z.L. Xiao, W.K. Kwok, D. Ray, C. Reichhardt, B. Jankó, Phys. C: Supercond. Appl. 533, 148 (2017)

47. D. Ray, C.J. Olson Reichhardt, B. Jankó, C. Reichhardt, Phys. Rev. Lett. 110, 267001 (2013)

48. Y.L. Wang, M.L. Latimer, Z.L. Xiao, R. Divan, L.E. Ocola, G.W. Crabtree, W.K. Kwok, Phys. Rev. B 87, 220501 (2013)

49. D. Ray, C. Reichhardt, C.J.O. Reichhardt, Phys. Rev. B 90, 094502 (2014)

50. V.V. Moshchalkov, M. Baert, V.V. Metlushko, E. Rosseel, M.J. Van Bael, K. Temst, Y. Bruynseraede, R. Jonckheere, Phys. Rev. B 57, 3615 (1998)

51. A.E. Koshelev, I.A. Sadovskyy, C.L. Phillips, A. Glatz, Phys. Rev. B 93, 060508 (2016)

52. N. Grønbech-Jensen, Comput. Phys. Commun. 119, 115 (1999)

53. J. Bardeen, M.J. Stephen, Phys. Rev. 140, A1197 (1965)

54. C. Tsallis, D.A. Stariolo, Physica A (1996)

55. N. Mangan, C. Reichhardt, C.J.O. Reichhardt, Phys. Rev. Lett. 100, 187002 (2008)

56. C.J. Olson, C. Reichhardt, R.T. Scalettar, G.T. Zimányi, N. Grønbech-Jensen, Phys. Rev. B 67, 184523 (2003) 